\title{
A pharmacokinetic model to document the actual disposition of topical ivermectin in cattle
}

\author{
Céline M. LAFFONT ${ }^{\mathrm{a}, \mathrm{b}}$, Alain BouSQUET-MÉLOU ${ }^{\mathrm{a} *}$, David BRALET ${ }^{\mathrm{a}}$, \\ Michel ALVINERIE ${ }^{\mathrm{c}}$, Johanna FINK-GREMMELS ${ }^{\mathrm{b}}$, Pierre-Louis TOUTAIN ${ }^{\mathrm{a}}$ \\ ${ }^{a}$ UMR 181 de Physiopathologie et Toxicologie Expérimentales INRA/ENVT, École Nationale \\ Vétérinaire de Toulouse, 23 Chemin des Capelles, 31076 Toulouse Cedex 03, France \\ ${ }^{b}$ Department of Veterinary Pharmacy, Pharmacology and Toxicology, Utrecht University, Yalelaan 16, \\ PO Box 80152, 3508 TD Utrecht, The Netherlands \\ ${ }^{\mathrm{c}}$ Institut National de la Recherche Agronomique, Station de Pharmacologie et Toxicologie, \\ 180 chemin de Tournefeuille, 31931 Toulouse Cedex, France
}

(Received 19 December 2002, accepted 25 March 2003)

\begin{abstract}
Ivermectin is a worldwide-used antiparasitic drug largely administered to cattle as a topical formulation (pour-on). The actual plasma and faecal disposition of pour-on ivermectin in cattle was documented using an original pharmacokinetic model, and taking into account the oral ingestion of the topical drug following physiological licking as a secondary route of exposure. Six pairs of monozygotic twin cattle received successively one i.v. and two pour-on administrations of ivermectin at a 3-5-month interval. For one pour-on administration, the twins were separated into an unrestrained group and a group where self- and allo-licking were prevented. Ivermectin concentrations in the plasma and faeces were determined by HPLC. Licking resulted in a high intraand inter-individual variability of systemic exposure after topical application. By the means of pharmacokinetic modelling, we showed that $58-87 \%$ of the pour-on dose was ingested, while only $10 \%$ was absorbed percutaneously. Approximately $72 \%$ of the ingested ivermectin transited directly into the faeces, resulting in a 7 -fold higher faecal excretion of the parent drug than in the non-lickers. We conclude that topical administration does not guarantee a controlled drug delivery in cattle. More importantly, the simulations revealed that non-treated cattle could get easily contaminated by allo-licking, raising the public health problem of unexpected drug residues in edible tissues.
\end{abstract}

ivermectin / pour-on / cattle / licking behaviour / pharmacokinetics

\section{INTRODUCTION}

Over the last twenty years, the topical route has received considerable interest in veterinary medicine for local and/or systemic delivery of drugs [12, 17, 22]. This application technique is particularly convenient for the owner who can easily apply the treatment himself with minimal risk of injury and minimal animal distress $[12,23]$. Furthermore, topical administration avoids the problems of hepatic first pass metabolism and of drug degradation in the gastrointestinal tract following oral

\footnotetext{
* Correspondence and reprints

Tel.: (33) 561193 925; fax: (33) 561193 917; e-mail: a.bousquet-melou@envt.fr
} 
administration, and therefore may be a good alternative to the oral route to achieve systemic therapeutic effects $[12,17]$.

Ivermectin is a worldwide-used antiparasitic drug, which is routinely administered to millions of cattle per year for systemic effects. As for other endectocides (doramectin, moxidectin, eprinomectin), the topical "pour-on" formulation of ivermectin has largely displaced the conventional injectable formulation in farming practices. In a recent study [15], we showed that the disposition of pour-on ivermectin in cattle was markedly modified when cattle licking behaviour was restricted. Licking is an important part of the natural grooming behaviour of animals in many species. In cattle, it serves an important physiological function in skin and hair hygiene, and plays a major role in the establishment and maintenance of the herd's social structure [14, $24,25,26]$. Prevention of licking in cattle results in a significantly lower absolute bioavailability of ivermectin, a two-times longer plasma elimination half-life, and a ten-times lower excretion of the parent drug in the faeces [15]. Altogether, these results suggest that following topical administration, a fraction of the applied dose of ivermectin reaches the systemic circulation by oral rather than dermal absorption, as a consequence of animal licking. The aim of the present study was thus to quantify the actual contribution of the oral route to the plasma and faecal disposition of topical ivermectin and to assess the resulting variability of exposure of topicallytreated cattle. For this purpose, an original pharmacokinetic model was developed. This model was applied to simulate the disposition of ivermectin under various conditions of oral and/or dermal exposure, addressing thereby the general problem of controlled drug delivery by topical application in animals. The consequences of animal social licking are discussed in terms of food safety objectives.

\section{MATERIALS AND METHODS}

\subsection{Study design and animals}

For the purpose of the study, a part of the raw data of our previous study [15] was used together with original data. Six pairs of monozygotic twin Holstein cattle (467 \pm $19 \mathrm{~kg}$ b.wt., 3 years old) obtained by micromanipulation [19] were used in the experiments. Each animal received a single i.v. administration of injectable ivermectin (IVOMEC ${ }^{\circledR}$ injectable, Merial, Lyon, France; $200 \mu \mathrm{g} / \mathrm{kg}$ b.wt.) and two topical administrations of ivermectin (IVOMEC ${ }^{\circledR}$ pour-on, Merial, Lyon, France) at the standard dose of $500 \mu \mathrm{g} / \mathrm{kg}$ b.wt. (PourOn 1 and Pour-On 2). All administrations were performed at a 3- to 5-month interval. For the second pour-on administration (Pour-On 2), the twins were separated into two groups of six animals. In the control group (the lickers), the animals were kept in individual tie-stalls, each cattle being tethered with a loose chain so that it could lick itself and its immediate neighbours. In the other group (the non-lickers), each animal was isolated from the others by a screen and was fitted with a wooden neck collar to prevent self-licking. Blood was collected regularly 17 times up to 28 days after application of Pour-On 1, 22 times up to 44 days after application of Pour-On 2, and 20 times up to 31 days following i.v. administration. Total faeces were collected during a period of 24 hours on days 4 and 14 after application of Pour-On 1. Following application of Pour-On 2, total faeces were collected for a period of $6 \mathrm{~h}$ (from 09:00 to $15: 00 \mathrm{~h}$ ) on days $1,2,3,4,7,14$, 18,22 and 28 post-administration.

\subsection{Analytical method}

The concentrations of ivermectin $(22,23$ dihydroavermectin B1a) in plasma and in wet faeces were measured using a high-performance liquid chromatography (HPLC) technique [2]. The lower limit of quantification for ivermectin was $0.05 \mathrm{ng} / \mathrm{mL}$ for 
the plasma and $0.5 \mathrm{ng}$ per $\mathrm{g}$ of wet faeces for the faecal samples. The limit of detection for ivermectin in plasma was $0.01 \mathrm{ng} / \mathrm{mL}$. Accuracy and precision (intra-assay variation) expressed as relative standard deviation were less than 8 and $6 \%$, respectively.

\subsection{Pharmacokinetic analysis}

\subsubsection{Non-compartmental approach}

Individual areas under the plasma concentration-time curve, $A U C s_{(0-i n f i n i t e)}$, were computed using the trapezoidal rule. The extrapolated area (from the last sample to infinity) was calculated by dividing the last plasma concentration measured by the slope of the terminal phase (estimated in a semi-logarithmic scale). Ivermectin (total) plasma clearance $\left(C L_{t o t}\right)$ was calculated as the ratio of the administered dose $(200 \mu \mathrm{g} / \mathrm{kg})$ divided by the $A U C_{(0 \text {-infinite) }}$ obtained for the i.v. route:

$$
C l_{\text {tot }}=\frac{\text { Dose }_{i, v .} .}{A U C_{i . v .}}
$$

The systemic availability for topical ivermectin $\left(F_{\text {tot }}\right)$ was calculated using the ratio of the $A U C s_{(0-i n f i n i t e)}$ obtained after topical $\left(A U C_{\text {pour-on }}\right)$ and i.v. $\left(A U C_{i . v}\right)$ administrations, corrected by the ratio of the administered doses:

$F_{\text {tot }}(\%)=\frac{A U C_{\text {pour-on }}}{A U C_{i . v .}} \times \frac{\text { Dose }_{i . v .}}{\text { Dose }_{\text {pour }-o n}} \times 100$.

Cumulative amounts of the parent ivermectin eliminated in the faeces were calculated by integration of the faecal excretion rate profile as a function of time, using the trapezoidal rule. The faecal excretion rates were obtained as the total amount of parent drug eliminated in the faeces within the collection interval (i.e., the product of the faecal concentration of ivermectin and the weight of wet faeces), divided by the time of collection.

\subsubsection{Data modelling}

Twin cattle were considered as the same animal taken under normal and restricted licking conditions. Each pair of twins was fitted separately. The pharmacokinetic model presented in Figure 1 was selected among other different models to simultaneously fit the plasma and faecal ivermectin data obtained in the licking and nonlicking twins after pour-on administration (Pour-On 2) and the i.v. plasma ivermectin data of the two twin cattle. The model was assumed dose-independent, but not timeindependent since the rate constant of ingestion of topical ivermectin $\left(k_{\mathrm{a}}\right)$ was described as a biexponential function of time. It was defined by the following equations:

$$
\begin{aligned}
C_{p}= & \frac{X_{1}}{V_{1}} \\
\frac{d X_{1}}{d t}= & -\left(k_{10}+k_{12}+k_{13}\right) \times X_{1}+k_{21} \times X_{2} \\
& +k_{31} \times X_{3}+k_{51} \times X_{5}+k_{61} \times X_{6}
\end{aligned}
$$

$\frac{d X_{2}}{d t}=k_{12} \times X_{1}-k_{21} \times X_{2}$

$\frac{d X_{3}}{d t}=k_{13} \times X_{1}-\left(k_{31}+k_{34}\right) \times X_{3}$

$\frac{d X_{4}}{d t}=k_{34} \times X_{3}+k_{64} \times X_{6}$

$\frac{d X_{5}}{d t}=-\left[k_{51}+k_{a}(t)+k_{57}\right] \times X_{5}$

$\frac{d X_{6}}{d t}=k_{a}(t) \times X_{5}-\left(k_{61}+k_{64}\right) \times X_{6}$

$\frac{d X_{7}}{d t}=k_{57} \times X_{5}$

where $C_{p}$ is the concentration of ivermectin in plasma (compartment 1 , volume $\left.V_{1}\right), X_{\mathrm{i}}$ is the amount of ivermectin in compartment $\mathrm{i}$, $k_{\mathrm{ij}}$ is the first-order rate constant of transfer from compartment $i$ to compartment $j$, and 


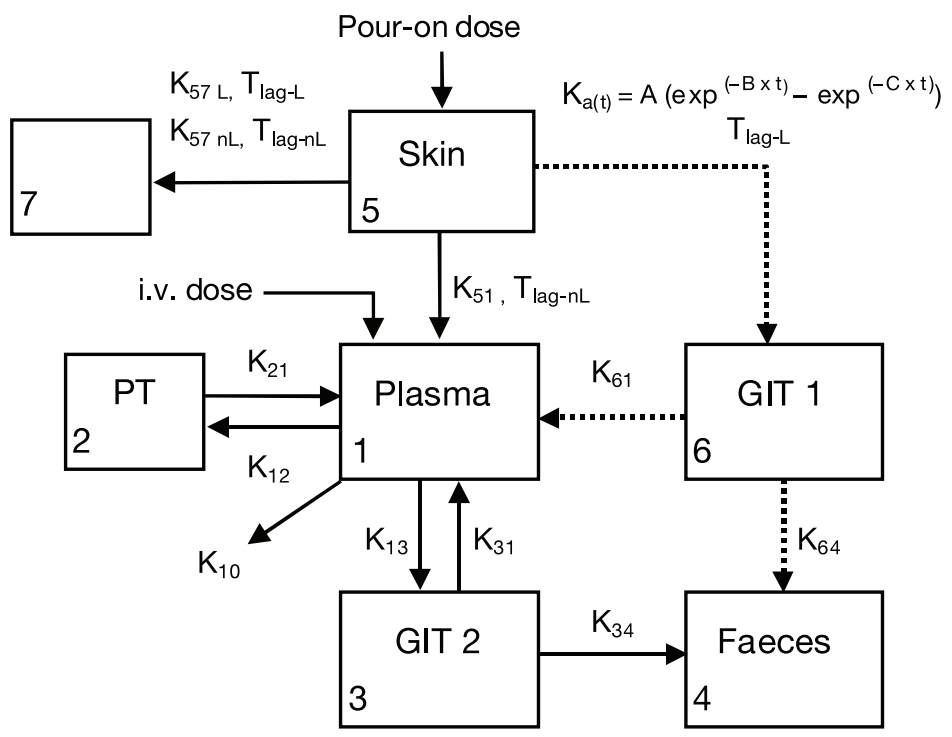

Figure 1. Disposition model for ivermectin after i.v. and topical (pour-on) administrations in one pair of licking and non-licking monozygotic twin cattle. $k_{\mathrm{ij}}$ is the first-order rate constant of transfer from compartment $\mathrm{i}$ to compartment $\mathrm{j} . k_{10}$ is the first-order rate constant of elimination from compartment 1 . The rate constant $k_{\mathrm{a}}$ transtates the ingestion of topical ivermectin by licking and is modelled as a biexponential function of time. The i.v. dose $(200 \mu \mathrm{g} / \mathrm{kg})$ is introduced in compartment 1 (plasma), while the pour-on dose $(500 \mu \mathrm{g} / \mathrm{kg}$ ) is introduced in compartment 5 (at the surface of the skin). The subscripts "L" and "nL" refer to the "licking" (control) or "non-licking" twin, respectively. "GIT l", gastrointestinal compartment 1. "GIT 2", gastrointestinal compartment 2. "PT", peripheral tissues.

$k_{10}$ is the first-order rate constant of elimination from compartment 1 .

For the disposition of topical ivermectin in non-lickers, $k_{\mathrm{a}}$ (the constant of transfer from compartment 5 to compartment 6) was set to zero. For the disposition of topical ivermectin in control animals (the lickers), $k_{\mathrm{a}}$ was empirically described as a function of time (Eq. 11), increasing up to a maximum and then decreasing (see discussion):

$k_{a}(t)=A \times[\exp (-B \times t)-\exp (-C \times t)]$

where the parameters $A, B$ and $C$ are expressed in $\mathrm{h}^{-1}$.

The i.v. dose $(200 \mu \mathrm{g} / \mathrm{kg})$ was introduced into the plasma compartment, while the pour-on dose $(500 \mu \mathrm{g} / \mathrm{kg})$ was intro- duced into compartment 5 representing the skin surface. $k_{12}, k_{21}, k_{13}, k_{31}$ are the distribution rate constants between the plasma and the peripheral compartments 2 and 3 . The rate constant $k_{10}$ relates to the elimination of ivermectin from plasma by metabolism, while the rate constant $k_{34}$ translates the removal of parent ivermectin from the gastrointestinal tract (GIT 2; compartment 3) into the faeces (compartment 4 ). $k_{51}$ is the rate constant for the systemic absorption of topical ivermectin via the skin. It was assumed that $k_{51}$ did not differ between lickers and non-lickers (see discussion). The rate constant $k_{57 \mathrm{~nL}}$ accounts for the fraction of topical ivermectin which was not systemically available by the dermal route in the non-licking cattle (e.g. left at the skin surface, photo-degraded, or 
metabolised by the enzymes of the skin). In control animals (the lickers), the topical drug could be absorbed through the skin $\left(k_{51}\right)$ or ingested by licking $\left(k_{\mathrm{a}}\right)$. One fraction of the ingested ivermectin was then absorbed in plasma $\left(k_{61}\right)$, while the remaining fraction transited unchanged into the faeces $\left(k_{64}\right)$. The model assumes that the local disposition of ivermectin is not the same at all sites of the digestive tract (see discussion). The rates of drug absorption and elimination were indeed different in the gastrointestinal compartments 3 (GIT 2) and 6 (GIT 1). In the lickers, the rate constant $k_{57 \mathrm{~L}}$ refers to the fraction of topical ivermectin which was neither ingested nor systemically-available by the dermal route. First pass metabolism of ivermectin by the oral route was considered negligible, given the low value of the drug total clearance (overall coefficient of extraction of $<1 \%$ in cattle). $T_{\text {lag-L }}$ is the delay to oral absorption, and $T_{\text {lag-nL }}$ is the delay to dermal absorption.

The differential equations were solved numerically using the SCIENTIST ${ }^{\circledR}$ programme (MicroMath ${ }^{\circledR}$ Scientific Software, Inc., Version 2.01). The goodness of fit was assessed by examination of the lines of best fit and of residual patterns, taking into account a modified Akaike information criterion. The data points were weighted by the inverse of the squared observed values (1/observation $\left.{ }^{2}\right)$ for ivermectin plasma concentrations, and by the inverse of the observed values (1/observation) for the amounts of ivermectin excreted in the faeces. The fitting was performed stepwise: (1) the i.v. plasma concentrations of ivermectin obtained in the two twins were fitted simultaneously to equations 3-6; (2) the i.v. plasma data of the twins and the pour-on (plasma and faecal) data of the non-licker were fitted simultaneously using equations 3-10 (the estimates yielded by the first fitting were used as initial values for the parameters); and (3) the pour-on (plasma and faecal) data of the licking and non-licking twins and the i.v. plasma data of the two cattle were fitted simultaneously using the equations 3-11. The estimates yielded by the second fitting were taken as initial values for the parameters.

Pharmacokinetic parameters were calculated from the final estimates of the model parameters. Ivermectin total (plasma) clearance, $C L_{\text {tot }}$, was calculated for each pair of twins according to the following equation (for a demonstration, see [18]):

$$
C l_{\text {tot }}=V_{1} \times\left(k_{10}+\frac{k_{34} \times k_{13}}{k_{31}+k_{34}}\right) .
$$

The relative importance of the dermal and oral routes in the removal of ivermectin from the skin was assessed for each licking cattle by calculating $X_{1}, X_{6}$ and $X_{7}$ using equations 4 and $8-10$ with $k_{10}, k_{12}$, $k_{13}, k_{61}, k_{64}$ equal to zero. The simulations were performed over a period of $6000 \mathrm{~h}$, until no ivermectin was left in compartment 5 . The fractions of topical ivermectin absorbed through the skin, ingested $\left(f_{\text {ingested }}\right)$, or remaining (left on the skin or degraded) were calculated as the final amount of ivermectin obtained by simulation in compartments 1,6 and 7, respectively, divided by the applied dose of the pour-on $(500 \mu \mathrm{g} / \mathrm{kg})$.

The absolute oral bioavailability of ivermectin $\left(F_{\text {oral }}\right)$ was estimated by modelling:

$$
F_{\text {oral }}(\%)=\frac{k_{61}}{k_{61}+k_{64}} \times 100 .
$$

The percentage of topical ivermectin systemically-available by the oral route was obtained as $f_{\text {ingested }} \times F_{\text {oral }}(\%)$.

\subsubsection{Simulation of the ingestion of topical ivermectin with increased rates of dermal absorption}

The influence of the rate of dermal absorption on the extent of drug ingestion was examined. Cumulative amounts of ivermectin ingested and absorbed through 
the skin after topical application were simulated for various magnitudes of dermal absorption rates. Simulations were performed using the estimated parameters obtained in each pair of twins. Equations 4 and $8-10$ were used for the simulations with $k_{10}, k_{12}, k_{13}, k_{61}, k_{64}$ equal to zero.

\subsubsection{Simulation of the exposure of non-treated animals following cross-contamination by allo-licking}

The possibility of the cross-contamination of non-treated cattle by licking of those topically-treated was examined. Simulations were carried out to evaluate the minimal amount of ivermectin that had to be ingested to achieve non-negligible plasma exposure of untreated cattle. Residues of ivermectin in milk were given as an indicator of the contamination of edible tissues. The simulations were performed for each pair of twins using the final estimated parameters. For simulation of a single oral uptake, the dose was introduced into compartment 6 . In the case of the ingestion of topical ivermectin by multiple oral uptake (represented by $k_{\mathrm{a}}$ ), the dose was introduced into compartment 5 with $k_{51}$ and $k_{57 \mathrm{~L}}$ equal to zero, and equations 3-9 and 11 were used for the simulations. Residues of ivermectin in milk were predicted from the corresponding plasma concentrations of ivermectin, given a milk/plasma concentration ratio of 0.766 [28].

\subsubsection{Statistical analysis}

Statistical analysis was performed using the SYSTAT ${ }^{\circledR} 8.0$ software (SPSS Inc., Chicago, IL). ANOVA was used to compare the within-pair and between-pair variability of plasma exposure $(A U C)$ and of ivermectin total clearance after i.v. administration in the 6 pairs of twins. ANOVA was also applied to compare the intra- and inter-individual variability of plasma exposure $(A U C)$ in the licker group after pour-on
A)

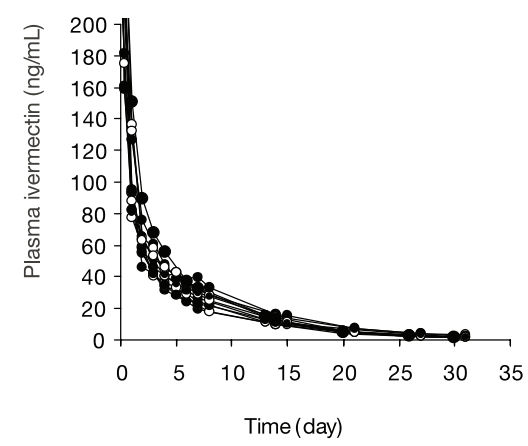

B)

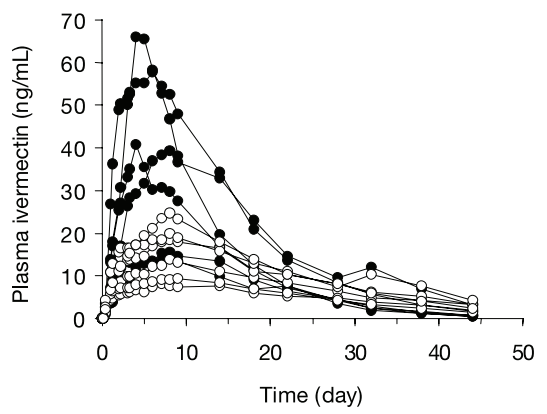

Figure 2. Ivermectin plasma concentrationtime curves in 6 pairs of monozygotic twin cattle after i.v. administration (A) and topical (pour-on) administration (B). The twins were separated in two groups of 6 animals: the licker group (filled symbols) and the non-licker group (open symbols). For the pour-on administration, self- and allo-licking were prevented in the cattle of the non-licker group.

administration. A $P<0.05$ was considered as significant. The results were expressed as the mean \pm standard deviation (SD).

\section{RESULTS}

\subsection{Intravenous administration of ivermectin}

The plasma concentration profiles of ivermectin obtained in the twelve cattle were very homogenous (Fig. 2A). It must be noted that for all pairs of twins, the 
A)

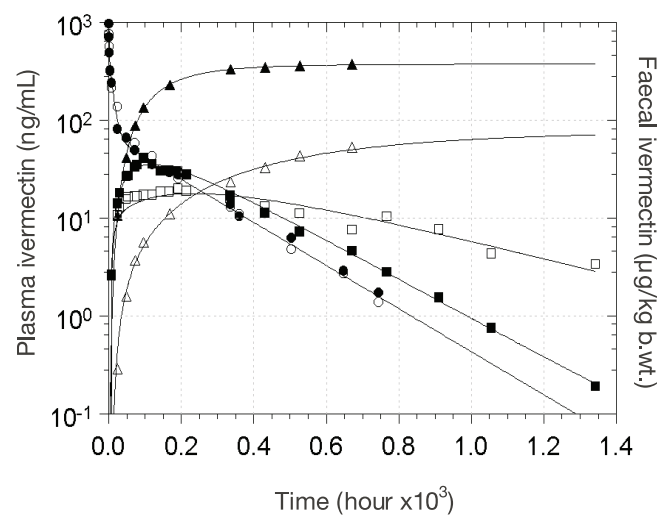

B)

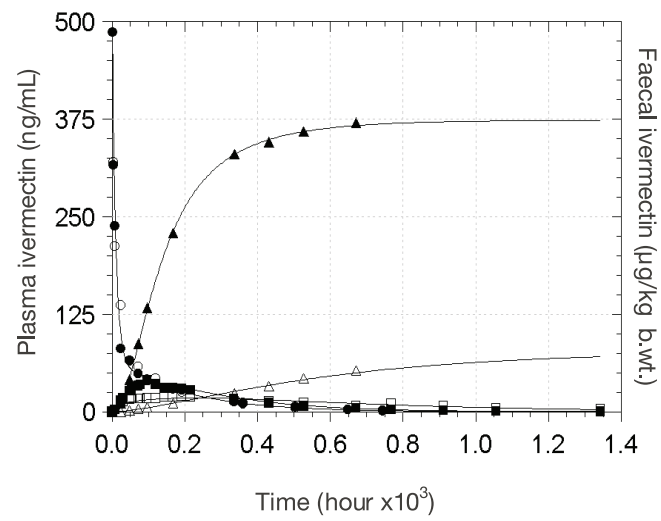

Figure 3. Experimental (symbols) and simulated (line) ivermectin data after i.v. and topical (pour-on) administrations in a representative pair of monozygotic twin cattle. Circle symbols, plasma concentrations of ivermectin after i.v. administration. Triangle symbols, cumulative amounts of parent ivermectin excreted in the faeces after topical administration. Square symbols, plasma concentrations of ivermectin after topical administration. Licking was prevented in one of the two twins (open symbols; non-licking cattle), the other twin serving as a control (filled symbols; licking cattle). (A) semi-logarithmic scale. (B) arithmetic scale. plasma concentration-time curves of the two twins were exactly superposed (see Fig. 3 for a representative pair of twins). Furthermore, the variability in plasma $A U C$ was significantly lower between the twins (coefficient of variation (CV) of 5\%) than that between the different pairs of twins $(\mathrm{CV}$ of $25 \%)(P<0.01)$. Similar results were obtained for ivermectin total clearance, with a lower within-pair than between-pair variability $(P<0.01)$. Altogether, these results consolidate the choice of using monozygotic twins for the purpose of modelling.

The i.v. plasma data were well fitted by the model, as it is shown for a representative pair of twin cattle in Figure 3. The individual total clearances of ivermectin estimated by modelling were consistent with those obtained by non-compartmental analysis (see Tab. I).

\subsection{Pour-On administration of ivermectin}

The plasma concentration-time profiles of ivermectin obtained after application of Pour-On 2 are presented in Figure 2B. By comparison with the i.v. route (Fig. 2A), the systemic exposure of animals was highly variable, depending on their ability to lick or not (CV of $55 \%$ in lickers vs. $29 \%$ in non-lickers).

All cattle showed a high variability of plasma exposure under normal licking conditions. After application of Pour-On 1, the difference in ivermectin bioavailability within the same pair of twins could be as 
Table I. Ivermectin total clearance $\left(C L_{t o t}\right)$ in cattle, and evaluation of the contribution of licking to the faecal excretion of parent ivermectin after topical application to cattle.

\begin{tabular}{|c|c|c|c|c|c|c|}
\hline \multirow[b]{2}{*}{ Pair number } & \multicolumn{2}{|c|}{$C L_{\text {tot }}(\mathrm{mL} / \mathrm{day} / \mathrm{kg})$} & \multicolumn{4}{|c|}{$\%$ dose eliminated unchanged in the faeces of lickers } \\
\hline & Non-comp. & Comp. & $\begin{array}{l}\text { Non-comp. } \\
\text { (0-28 days) }\end{array}$ & $\begin{array}{c}\text { Comp. } \\
\text { (0-28 days) }\end{array}$ & $\begin{array}{c}\text { Comp. } \\
(0 \text { infinite })\end{array}$ & $\begin{array}{l}\text { By the oral } \\
\text { route }\end{array}$ \\
\hline \multicolumn{7}{|l|}{ Non-lickers } \\
\hline 1 & 230.8 & 227.9 & 6.5 & 6.7 & 9.0 & - \\
\hline 2 & 286.0 & 322.9 & 3.3 & 4.3 & 6.4 & - \\
\hline 3 & 332.7 & 325.1 & 5.7 & 5.3 & 7.1 & - \\
\hline 4 & 283.6 & 296.3 & 6.9 & 4.6 & 6.5 & - \\
\hline 5 & 236.7 & 258.2 & 10.6 & 10.0 & 15.5 & - \\
\hline 6 & 225.6 & 233.0 & 6.8 & 7.7 & 10.2 & - \\
\hline Mean \pm SD & $266 \pm 42.2$ & $277 \pm 43.6$ & $6.6 \pm 2.3$ & $6.4 \pm 2.2$ & $9.1 \pm 3.5$ & - \\
\hline \multicolumn{7}{|l|}{ Lickers } \\
\hline 1 & 211.1 & 227.9 & 74.2 & 74.8 & 80.5 & 59.4 \\
\hline 2 & 322.1 & 322.9 & 76.0 & 75.4 & 77.5 & 70.4 \\
\hline 3 & 328.3 & 325.1 & 55.0 & 54.2 & 56.5 & 48.2 \\
\hline 4 & 355.5 & 296.3 & 83.2 & 54.0 & 55.7 & 44.0 \\
\hline 5 & 214.4 & 258.2 & 74.1 & 73.4 & 74.8 & 61.3 \\
\hline 6 & 210.2 & 233.0 & 53.6 & 56.8 & 57.6 & 43.9 \\
\hline Mean \pm SD & $274 \pm 68.5$ & $277 \pm 43.6$ & $69 \pm 12.1$ & $65 \pm 10.7$ & $67 \pm 11.6$ & $55 \pm 10.8$ \\
\hline
\end{tabular}

Compartmental (comp.) and non-compartmental (non-comp.) analyses were performed in the 6 pairs of monozygotic twin cattle.

high as $70 \%$. In the licker group, the plasma $A U C$ of a same animal could be multiplied by a factor of 0.6 to 2.3 from one pour-on application to the other (PourOn 1 vs. Pour-On 2). The intra-individual variability of exposure (CV of $41 \%$ ) was not significantly lower than the inter-individual variability (CV of $55 \%$ ).

There was a major difference in the faecal excretion of the parent drug between licking and non-licking animals (Fig. 4A and Tab. I). For the non-licker group, it was possible to compare the disposition of pour-on ivermectin in the same animal under normal (Pour-On 1) and restricted (Pour-On 2) licking conditions. As shown in Figure 4B, the faecal excretion rates observed in these cattle after application of Pour-On 1 were much higher than the following application of Pour-On 2 and matched those found in their twins (licker group) following the administration of Pour-On 1 and Pour-On 2.

The plasma and faecal data obtained for topical ivermectin were all well fitted by the model (Tab. I; see Fig. 3 for a representative pair of twins). In one pair of twins however, the predicted faecal data underestimated by $35 \%$ the amounts of the parent ivermectin actually excreted in the faeces of the licker, which will be further discussed. The absolute bioavailabilities $\left(F_{\text {tot }}\right)$ of pour-on ivermectin estimated by modelling were very close to those obtained by non-compartmental analysis in lickers and non-lickers (Tab. II). The estimated parameters of the overall disposition model presented in Figure 1 are listed in Table III. It is noteworthy that the parameters showing the highest coefficients of variation were those associated with the ingestion of the topical drug by licking $\left(A, C, k_{61}, k_{64}, k_{57 \mathrm{~L}}, T_{\text {lag-L }}\right)$. 
A)

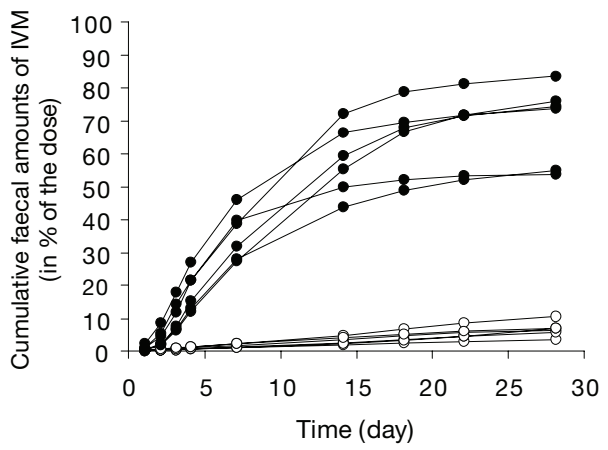

B)

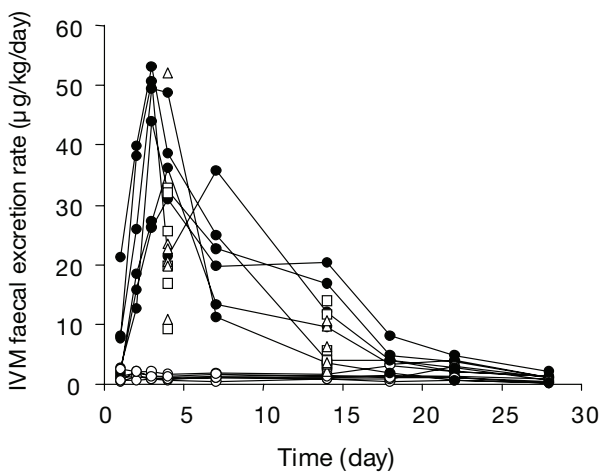

Figure 4. Faecal excretion of parent ivermectin (IVM) following topical application of 6 pairs of monozygotic twin cattle (Pour-On 2). Twins were separated into two groups of 6 animals: the non-licker group (open circles), in which self-and allo-licking were prevented, and the licker group (filled circles, control group). (A) Cumulative amounts of ivermectin excreted in the faeces. (B) Ivermectin faecal excretion rates. Open triangles and open squares refer to a previous pour-on administration (Pour-On 1) in the same animals (licker and nonlicker groups, respectively), with this time no restriction of licking in any of the two groups.

Table II. Absolute bioavailability $\left(F_{t o t}\right)$ of pour-on ivermectin in cattle, and evaluation of the extent of ingestion, oral absorption, and dermal absorption of ivermectin after topical application.

\begin{tabular}{|c|c|c|c|c|c|c|}
\hline \multirow[b]{2}{*}{ Pair number } & \multicolumn{2}{|c|}{$F_{t o t}(\%)$} & \multicolumn{4}{|c|}{$\%$ of the dose } \\
\hline & $\begin{array}{l}\text { Non-comp. } \\
\text { analysis }\end{array}$ & $\begin{array}{c}\text { Comp. } \\
\text { analysis }\end{array}$ & Ingested & $\begin{array}{c}\text { Absorbed } \\
\text { orally }\end{array}$ & $\begin{array}{c}\text { Absorbed } \\
\text { through the skin }\end{array}$ & Left \\
\hline \multicolumn{7}{|l|}{ Non-lickers } \\
\hline 1 & 17.4 & 16.2 & - & - & 16.2 & 83.8 \\
\hline 2 & 15.6 & 16.2 & - & - & 16.2 & 83.8 \\
\hline 3 & 16.9 & 17.5 & - & - & 17.5 & 82.5 \\
\hline 4 & 30.1 & 31.0 & - & - & 31.0 & 69.0 \\
\hline 5 & 29.9 & 32.8 & - & - & 32.8 & 67.2 \\
\hline 6 & 24.2 & 23.2 & - & - & 23.2 & 76.8 \\
\hline Mean \pm SD & $22 \pm 6.6$ & $23 \pm 7.5$ & - & - & $23 \pm 7.5$ & $77 \pm 7.5$ \\
\hline \multicolumn{7}{|l|}{ Lickers } \\
\hline 1 & 35.6 & 37.8 & 83.4 & 24.0 & 13.8 & 2.6 \\
\hline 2 & 18.2 & 18.0 & 80.5 & 10.1 & 7.9 & 11.7 \\
\hline 3 & 21.4 & 20.4 & 58.4 & 10.2 & 10.2 & 31.4 \\
\hline 4 & 68.4 & 56.0 & 86.6 & 42.6 & 13.4 & 0.0 \\
\hline 5 & 22.9 & 28.4 & 80.1 & 18.7 & 9.7 & 10.3 \\
\hline 6 & 29.6 & 31.1 & 67.8 & 23.9 & 7.3 & 25.0 \\
\hline Mean \pm SD & $33 \pm 18.6$ & $32 \pm 13.8$ & $76 \pm 10.8$ & $22 \pm 12.0$ & $10 \pm 2.7$ & $13 \pm 12.4$ \\
\hline
\end{tabular}

Compartmental (comp.) and non-compartmental (non-comp.) analyses were performed in the 6 pairs of monozygotic twin cattle. 
Table III. Estimated parameters of the seven-compartment model (Fig. 1) selected to describe the i.v and topical disposition of ivermectin in cattle.

\begin{tabular}{|c|c|c|c|c|c|c|c|c|}
\hline \multirow[b]{2}{*}{ Parameters } & \multicolumn{6}{|c|}{ Pair number } & \multirow[b]{2}{*}{ Mean } & \multirow[b]{2}{*}{$\% \mathrm{CV}$} \\
\hline & 1 & 2 & 3 & 4 & 5 & 6 & & \\
\hline$V_{1}(\mathrm{~L} / \mathrm{kg})$ & 0.078 & 0.084 & 0.080 & 0.093 & 0.074 & 0.058 & 0.078 & 15 \\
\hline$k_{10}\left(\mathrm{~h}^{-1}\right)$ & 0.054 & 0.096 & 0.101 & 0.105 & 0.077 & 0.094 & 0.088 & 22 \\
\hline$k_{12}\left(\mathrm{~h}^{-1}\right)$ & 0.288 & 0.293 & 0.439 & 0.271 & 0.283 & 0.358 & 0.322 & 20 \\
\hline$k_{21}\left(\mathrm{~h}^{-1}\right)$ & 0.016 & 0.016 & 0.019 & 0.020 & 0.017 & 0.021 & 0.018 & 13 \\
\hline$k_{64}\left(\mathrm{~h}^{-1}\right)$ & 0.345 & 0.057 & 0.038 & 1.929 & 0.145 & 0.021 & 0.423 & 177 \\
\hline$k_{61}\left(\mathrm{~h}^{-1}\right)$ & 0.140 & 0.008 & 0.008 & 1.868 & 0.044 & 0.011 & 0.347 & 216 \\
\hline$k_{57 \mathrm{~L}}\left(\mathrm{~h}^{-1}\right)$ & 0.00011 & 0.00058 & 0.00181 & 0.00000 & 0.00071 & 0.00223 & 0.00091 & 101 \\
\hline$k_{57 \mathrm{~nL}}\left(\mathrm{~h}^{-1}\right)$ & 0.00269 & 0.00192 & 0.00245 & 0.00176 & 0.00143 & 0.00216 & 0.00207 & 22 \\
\hline$k_{13}\left(\mathrm{~h}^{-1}\right)$ & 1.760 & 3.872 & 3.693 & 2.096 & 1.673 & 3.242 & 2.723 & 37 \\
\hline$k_{31}\left(\mathrm{~h}^{-1}\right)$ & 0.642 & 0.883 & 0.918 & 0.484 & 0.491 & 0.889 & 0.718 & 28 \\
\hline$k_{34}\left(\mathrm{~h}^{-1}\right)$ & 0.026 & 0.015 & 0.018 & 0.006 & 0.021 & 0.021 & 0.018 & 37 \\
\hline$T_{\text {lag-L }}(\mathrm{h})$ & 37.1 & 14.3 & 27.4 & 20.0 & 0.0 & 5.4 & 17.4 & 79 \\
\hline$T_{\text {lag-nL }}(\mathrm{h})$ & 6.8 & 5.4 & 5.0 & 2.0 & 7.1 & 5.7 & 5.3 & 35 \\
\hline$A\left(\mathrm{~h}^{-1}\right)$ & 0.0186 & 0.0480 & 0.0042 & 0.0093 & 0.0108 & 0.0114 & 0.0170 & 93 \\
\hline$B\left(\mathrm{~h}^{-1}\right)$ & 0.00325 & 0.00215 & 0.00096 & 0.00179 & 0.00158 & 0.00241 & 0.00202 & 39 \\
\hline$C\left(\mathrm{~h}^{-1}\right)$ & 0.00731 & 0.00308 & 0.04622 & 0.02854 & 0.01658 & 0.02418 & 0.02098 & 75 \\
\hline$k_{51}\left(\mathrm{~h}^{-1}\right)$ & 0.00052 & 0.00037 & 0.00052 & 0.00079 & 0.00070 & 0.00065 & 0.00059 & 25 \\
\hline
\end{tabular}

Plasma (i.v., pour-on) and faecal (pour-on) data of six pairs of monozygotic twin cattle were used for the compartmental analysis. Twins were separated as "lickers" and "non-lickers": licking was prevented in one twin, the other twin serving as a control. Each pair of twins was fitted separately. "L", lickers; "nL", non-lickers.

The model revealed that $58-87 \%$ of the topically-applied ivermectin was actually ingested by licking $\left(f_{\text {ingested }}\right.$, Tab. II). A comparison of the amounts of drug absorbed by the oral and dermal routes in lickers indicates that $50-77 \%$ of ivermectin systemic absorption was achieved by the oral route vs. $23-50 \%$ by the dermal route (Tab. II). The oral absolute bioavailability of ingested ivermectin $\left(F_{\text {oral }}\right)$ was estimated by modelling $(28 \pm 13.2 \%$; Eq. 13) and tended to be higher than for the dermal route in both control and non-licking cattle (Tab. II). Together with the large ingestion of the topical drug, this explains the higher overall bioavailability of pouron ivermectin $\left(F_{t o t}\right)$ in lickers compared to non-licking cattle (see Tab. II). It results from the determination of $F_{\text {oral }}$ that approximately $72 \%$ of the ingested ivermectin was not absorbed and transited directly into the faeces. This fraction represented approximately $55 \%$ of the applied dose $\left(f_{\text {ingested }} \times\left(1-F_{\text {oral }}\right) ;\right.$ Tab. I $)$ and accounted for $82 \%$ of the overall excretion of the parent drug in the dung $(67 \%$ of the dose; Tab. I). This explains why the faecal excretion of parent ivermectin 


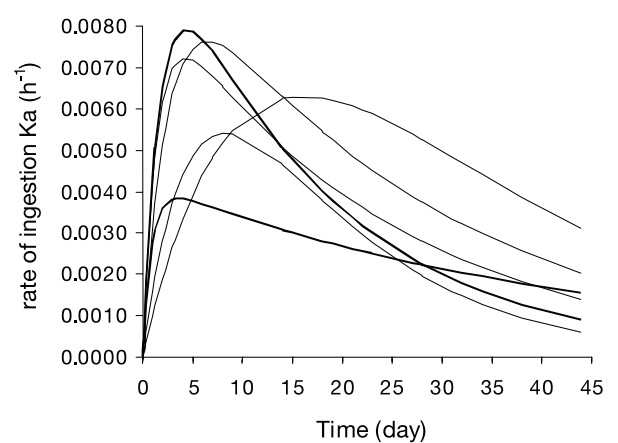

Figure 5. The rate constant of ingestion, $k_{\mathrm{a}}$, obtained by modelling is plotted as a function of time, for each of the 6 licking cattle.

(extrapolated to infinity) was 7-times higher in lickers than in non-lickers (Tab. I).

Individual time profiles of the ingestion rate constant, $k_{\mathrm{a}}$, were generated by modelling and are presented in Figure 5. Peak values of $k_{\mathrm{a}}$ were reached between 3 and 16 days after pour-on application. Half of the ingestion was achieved between 3 and 7 days post-administration, and $90 \%$ of the ingestion was achieved between 9 and 17 days post-administration. The differences observed among cattle are consistent with the large variability expected from animal licking.

Ingestion profiles were simulated for different magnitudes of dermal absorption rates using the final set of estimated parameters obtained for each pair of twins (Tab. III). The simulations indicate that the rate constant of dermal absorption, $k_{51}$, must be increased by a factor of 6-10 to have less ivermectin ingested by licking than that absorbed through the skin, and by a factor of at least 50 to have a 10-times lower ingestion of ivermectin (see Fig. 6 for a representative pair of twin cattle). An 8 -fold increase in $k_{51}$ resulted in a 5-times higher bioavailability of ivermectin via the skin (48 $\pm 6.4 \%$ of the dose instead of $10 \pm 2.7)$ and in lower amounts of ivermec- tin remaining as a cutaneous depot or degraded $(8 \pm 7.4 \%$ of the dose instead of $13 \pm 12.4)$.

\subsection{Simulation of the contamination of non-treated animals with ivermectin by allo-licking}

Our simulations indicate that in nontreated cattle, a continuous licking (simulated by $k_{\mathrm{a}}$ ) accounting for $2-11 \%$ of the pour-on dose in total would be sufficient to achieve ivermectin plasma concentrations of $1 \mathrm{ng} / \mathrm{mL}$ (see Tab. IV), along with detectable concentrations of ivermectin in the milk for 44 days (maximal concentration of $0.8 \mathrm{ng} / \mathrm{mL}$ ). Detectable concentrations in plasma $(0.01 \mathrm{ng} / \mathrm{mL})$ would be achieved by ingestion of 100-times lower amounts $(0.02-0.11 \%$ of the dose in total).

In the case of a single oral uptake, ingestion of $0.3-2.5 \%$ of the pour-on dose would be sufficient to achieve plasma concentrations of $1 \mathrm{ng} / \mathrm{mL}(0.8 \mathrm{ng} / \mathrm{mL}$ in milk) (Tab. IV). Detectable concentrations of ivermectin in plasma would be achieved if non-treated cattle licked $0.003-0.025 \%$ of the pour-on dose.

\section{DISCUSSION}

In contrast to what could be expected for a "topical" formulation, the present study shows that the main route for the systemic absorption of topical ivermectin in cattle is not dermal but oral, as a result of cattle licking behaviour. This is the first time such an interaction between the individual/social behaviour of animals and the pharmacokinetics of parenterally-administered drugs is reported.

In order to evaluate the extent of ivermectin absorption by the dermal and oral routes, a modelling approach was required together with the use of monozygotic twin cattle, each pair of twins being considered as a single animal. The selected pharmacokinetic model adequately describes the 
a)

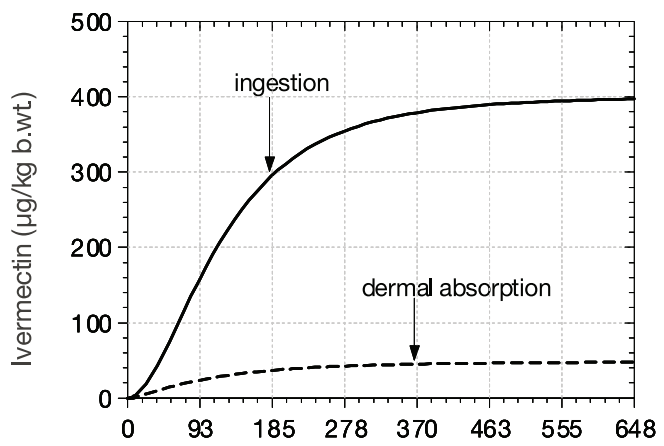

b)

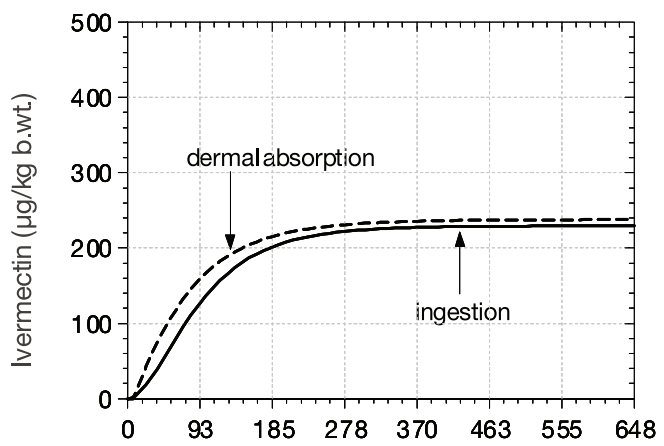

c)

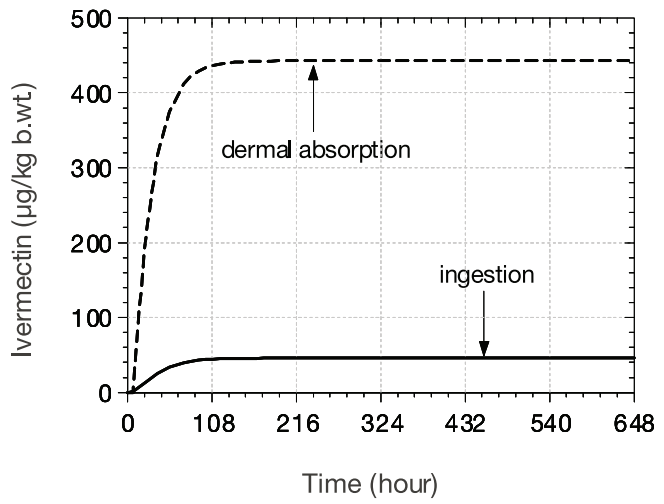

Figure 6. Simulations of the dermal absorption (medium-dashed line) and ingestion (solid line) of topical ivermectin with increased rates of dermal absorption in one representative licking cattle. (a) Control values. (b) Dermal absorption rate multiplied by 8 . (c) Dermal absorption rate multiplied by 50 . experimental plasma and faecal data observed after i.v. and Pour-On administration of ivermectin in both licking and non-licking cattle. The predicted values for the absolute bioavailability of pour-on ivermectin $\left(F_{t o t}\right)$ and for the drug total clearance $\left(C L_{t o t}\right)$ were very close to those obtained by non-compartmental analysis. It was assumed that the rate constant of percutaneous absorption, $k_{51}$, did not differ between lickers and non-lickers. This was supported by a non-significant difference in the estimates of $k_{51 \mathrm{~L}}$ and $k_{51 \mathrm{~nL}}$ when considered as separate parameters in the model (data not shown), which suggests that the absence of licking for 44 days did not impair the process of ivermectin absorption through the skin. 
Table IV. Simulation of the contamination of non-treated cattle, which would lick those topicallytreated. Simulations of a single or multiple oral uptake were performed for each pair of twins, using the final estimated parameters (listed in Tab. III).

\begin{tabular}{lcc}
\hline & \multicolumn{2}{c}{$\begin{array}{c}\text { \% dose which needs to be ingested to reach plasma concentrations } \\
\text { of } 1 \mathrm{ng} / \mathrm{mL} \text { in non-treated cattle }\end{array}$} \\
\cline { 2 - 3 } Pair number & Multiple oral uptake (continuous licking) & Single oral uptake \\
\hline 1 & 3.6 & 0.3 \\
2 & 11.1 & 2.5 \\
3 & 10.0 & 2.3 \\
4 & 2.0 & 2.0 \\
5 & 3.7 & 0.6 \\
6 & 2.1 & 0.8 \\
\hline
\end{tabular}

It is worthwhile to mention that the pour-on data obtained in lickers were totally misfitted by a model containing a single gastrointestinal compartment pooling proximal (GIT 1) and distal (GIT 2) digestive compartments. Selection of a model with two separate gastrointestinal compartments suggests that the local disposition of ivermectin is not the same at all sites of the gastrointestinal tract in terms of (re-)absorption and elimination. This is in line with the experimental finding of a different bioavailability of ivermectin in the rumen and in the abomasum in sheep (75\% lower after intraruminal administration than after intra-abomasal administration) [21]. This difference in bioavailability was first attributed to an extensive degradation or metabolisation of ivermectin in the rumen [21]. This explanation, however, is difficult to conciliate with our results, since the unabsorbed fraction of ingested ivermectin was fully recovered in the faeces of the lickers. Moreover, several studies argue for the stability of ivermectin in rumen fluids in cattle and sheep [3,6] and show that there is rather an extensive adsorption of ivermectin to the digesta particulates of the rumen [1]. Our model stipulates that the fraction of drug which is not absorbed in compartment GIT 1 will not be absorbed later in compartment GIT 2. A possible interpretation is that ivermectin remains adsorbed to the particulate phase of the digesta during intestinal transit, and thus is not available anymore for absorption in the intestinal fluids. Such a situation has already been described for other compounds such as phenylbutazone $[5,16]$ and is consistent with the high organic-carbon binding constant of ivermectin $\left(\mathrm{K}_{\mathrm{oc}}=\right.$ 12600-15700; [11]) and its high hydrophobicity [20]. Ivermectin oral bioavailability was estimated from the model to be $28 \pm$ $13.2 \%$, which is in line with the bioavailability found by Chiu et al. [8] in cattle for an intraruminal bolus of ivermectin relative to the subcutaneous route (26\%). However, the contribution of a buccal absorption of ivermectin cannot be excluded.

Pharmacokinetic modelling showed that approximately $76 \%$ of the pour-on dose was ingested by licking. As indicated by preliminary deconvolution studies, the rate constant of ingestion, $k_{\mathrm{a}}$, could be described as a biexponential function of time, with an increase up to a maximum followed by a gradual decrease. As shown for the antiparasitic agents cypermethrin [13], flumethrin [27] and parathion [7] in sheep, cattle or pigs, it is possible that ivermectin spreads over the skin of cattle. The first increase of $k_{\mathrm{a}}$ would then be explained by a higher licking efficiency, due to a progressive increase in skin surface exposure. Conversely, the decrease of $k_{\mathrm{a}}$ would 
correspond to a reduction in licking efficiency, consistent with the decrease of ivermectin concentrations on the skin. Kinetic modelling shows an ingestion of topical ivermectin up to 19 days postadministration, suggesting that ivermectin was available at the skin surface for a long period of time. This is in agreement with previously published results, suggesting that ivermectin is still present on the skin of non-licking cattle 44 days post-administration [15].

The model indicates that $50-77 \%$ of ivermectin absorption in plasma was achieved by the oral route, compared with $23-50 \%$ by the dermal route. This infers that the systemic exposure of animals depends most likely on their ability to lick themselves (self-licking) or each other (allo-licking). This would explain the erratic bioavailability of pour-on ivermectin observed in the licker group and in previous studies [9], as well as the large intraindividual and within-pair variability of plasma exposure (up to $230 \%$ and $70 \%$, respectively), which cannot be of genetic origin. More generally, the extent of licking depends on various social, nutritional, physiological, pathological, environmental and managerial factors [24, 25], which makes the delivery of the drug even more unpredictable. An increased licking activity has been reported in parasitised cattle [24], which could be in favour of a higher plasma exposure of animals and a better efficacy of the pour-on treatment. On the other hand, a lower exposure of animals could result in subtherapeutic plasma concentrations, which may promote the development of drug resistance [10]. Finally, the results question the relevance of performing in vivo bioequivalence assays for the evaluation of topical drug formulations in licking animals.

For simplicity reasons, allo-licking was not taken into account in the model. However, although the cattle of the licking group were housed in individual boxes, contacts by allo-licking were observed during the experiments between the immediate neighbours. In one pair of twins, the pour-on dose $(500 \mu \mathrm{g} / \mathrm{kg})$ applied on the back of the licker did not provide a sufficient amount of ivermectin to explain the high faecal data (no ivermectin was left on the skin). This suggests a supplementary source of topical drug consistent with allolicking.

Under field conditions, allo-licking in cattle may result in the contamination of non-treated animals following contact with topically treated cattle. Our simulations (multiple oral uptake) showed a significant (detectable) plasma exposure of nontreated animals, which would lick small amounts of ivermectin from the skin of one or several treated cattle $(0.02-0.11 \%$ of the pour-on dose in total). It is noteworthy that a continuous licking accounting for $2-11 \%$ of the pour-on dose in total would result in detectable concentrations in the milk of non-treated cattle for more than 44 days with maximal plasma concentrations of $1 \mathrm{ng} / \mathrm{mL}$. These ingested amounts depend obviously on the individual licking activity (translated by the $k_{\mathrm{a}}$ function), but are all very low compared to the $76 \%$ of the dose actually ingested by the treated cattle under the same conditions. Altogether, these results suggest that a non-negligible contamination of non-treated cattle is likely to occur following social contact with the treated animals. This elicits concern over possible and unpredictable insecticide residues in the milk and edible tissues of such animals.

The skin provides an excellent barrier against the environment and foreign substances, and many drugs used in human and veterinary medicine exhibit a low rate and extent of dermal absorption $[4,17,22]$. In the present study however, we show that a low rate of absorption through the skin may not ensure a controlled and optimised delivery of drug to the systemic or local circulation. Licking behaviour reduced by a factor of two the total amount of ivermectin delivered to the plasma by the dermal 
route (from $20 \%$ of the dose in non-lickers to $10 \%$ in lickers) and led to an unpredictable absorption of topical drug by the oral route. As indicated by the simulations, the rate constant of dermal absorption $\mathrm{k}_{51}$ would need to be increased by a factor of at least 50 to warrant a good dermal absorption of the drug, independently of animal licking behaviour or other external events (evaporation, accidental removal of the drug, ...).

In conclusion, we present an original pharmacokinetic model allowing the assessment of the oral uptake of drugs administered cutaneously to licking animals. This modelling approach revealed that the ingestion of ivermectin by licking constitutes a possible route of cross-contamination between animals, and thus has to be considered as a general risk inherent to the use of topical drug formulations in food-producing animals.

\section{REFERENCES}

[1] Ali D.N., Hennessy D.R., The effect of level of feed intake on the pharmacokinetic disposition and efficacy of ivermectin in sheep, J. Vet. Pharmacol. Ther. 19 (1996) 89-94.

[2] Alvinerie M., Sutra J.F., Galtier P., Toutain P.L., Determination of ivermectin in milk by high performance liquid chromatography, Ann. Rech. Vet. 18 (1987) 269-274.

[3] Andrew N.W., Halley B.A., Stability of ivermectin in rumen fluids, J. Vet. Pharmacol. Ther. 19 (1996) 295-299.

[4] Barry B.W., Novel mechanisms and devices to enable successful transdermal drug delivery, Eur. J. Pharm. Sci. 14 (2001) 101-114.

[5] Bogan J.A., Galbraith A., Baxter P., Ali N.M., Marriner S.E., Effect of feeding on the fate of orally administered phenylbutazone, trimethoprim and sulphadiazine in the horse, Vet. Rec. 115 (1984) 599-600.

[6] Bogan J.A., McKellar Q.A., The pharmacodynamics of ivermectin in sheep and cattle, $\mathrm{J}$ Vet. Pharmacol. Ther. 11 (1988) 260-268.

[7] Brimer L., Gyrd-Hansen N., Rasmussen F., Disposition of parathion after dermal application in pigs, J. Vet. Pharmacol. Ther. 17 (1994) 304-308.
[8] Chiu L., Green M.L., Baylis F.P., Eline D., Rosegay A., Meriwether H., Jacob T.A., Absorption, tissue distribution and excretion of tritium-labeled ivermectin in cattle, sheep and rat, J. Agr. Food Chem. 38 (1990) 20722078.

[9] Gayrard V., Alvinerie M., Toutain P.L., Comparison of pharmacokinetic profiles of doramectin and ivermectin pour-on formulations in cattle, Vet. Parasitol. 81 (1999) 47-55.

[10] Geerts S., Gryseels B., Drug resistance in human helminths: Current situation and lessons from Livestock, Clin. Microbiol. Rev. 13 (2000) 207-222.

[11] Halley B.A., Nessel R.J., Lu A.Y.H., Environmental aspects of ivermectin usage in livestock: general considerations, in: Campbell W.C. (Ed.), Ivermectin and Abamectin, New York Springer, 1989, pp. 162-172.

[12] Hennessy D.R., Modifying the formulation or delivery mechanism to increase the activity of anthelmintic compounds, Vet. Parasitol. 72 (1997) 367-382.

[13] Jenkinson D.M., Hutchison G., Jackson D., McQueen L., Route of passage of cypermethrin across the surface of sheep skin, Res. Vet. Sci. 41 (1986) 237-241.

[14] Krohn C.C., Behaviour of dairy cows kept in extensive (loose housing/pasture) or intensive (tie-stall) environments. III. Grooming, exploration and abnormal behaviour, Appl. Anim. Behav. Sci. 42 (1994) 73-86.

[15] Laffont C.M., Alvinerie M., Bousquet-Mélou A., Toutain P.L., Licking behaviour and environmental contamination arising from pouron ivermectin for cattle, Int. J. Parasitol. 31 (2001) 1687-1692.

[16] Lees P., Taylor J.B., Higgins A.J., Sedgwick A.D., In vitro and in vivo binding of phenylbutazone and related drugs to equine feeds and digesta, Res. Vet. Sci. 44 (1988) 50-56.

[17] Magnusson B.M., Kenneth A., Roberts M.S., Veterinary drug delivery: potential for skin penetration enhancement, Adv. Drug Deliver. Rev. 50 (2001) 205-227.

[18] Nakashima E., Benet L.Z., General treatment of mean residence time clearance, and volume parameters in linear mammillary models with elimination from any compartment, $\mathrm{J}$. Pharmacokinet. Biopharm. 16 (1988) 475492.

[19] Ozil J.P., Heyman Y., Renard J.P., Production of monozygotic twins by micromanipulation and cervical transfer in the cow, Vet. Rec. 110 (1982) 126-127. 
[20] Pouliot J.F., L'Heureux F., Liu Z., Prichard R.K., Georges E., Reversal of P-glycoprotein-associated multidrug resistance by ivermectin, Biochem. Pharmacol. 10 (1997) $17-25$.

[21] Prichard R.K., Steel J.W., Lacey E., Hennessy D.R., Pharmacokinetics of ivermectin in sheep following intravenous, intraabomasal or intraruminal administration, J. Vet. Pharmacol. Ther. 8 (1985) 88-94.

[22] Riviere J.E., Papich M.G., Potential and problems of developing transdermal patches for veterinary applications, Adv. Drug Deliver. Rev. 50 (2001) 175-203.

[23] Rothen-Weinhold A., Gurny R., Dahn M. Formulation and technology aspects of controlled drug delivery in animals, Pharm. Sci. Technol. Today 3 (2000) 222-231.

[24] Sato S., Sako S., Maeda A., Social licking patterns in cattle (Bos taurus): influence of environmental and social factors, Appl. Anim. Behav. Sci. 32 (1991) 3-12.

[25] Sato S., Tarumizu K., Hatae K., The influence of social factors on allogrooming in cows, Appl. Anim. Behav. Sci. 38 (1993) 235-244.

[26] Simonsen H.B., Grooming behaviour of domestic cattle, Nord. Vet. Med. 31 (1979) $1-5$.

[27] Stendel W., Hamel H.D., Sieveking H.U., Bruhne D., Analytical determination of the distribution of flumethrin on the body surface of cattle following topical pour-on application, Vet. Parasitol. 42 (1992) 137-143.

[28] Toutain P.L., Campan M., Galtier P., Alvinerie M., Kinetic and insecticidal properties of ivermectin residues in the milk of dairy cows, J. Vet. Pharmacol. Ther. 11 (1988) 288-291. 